\title{
Case Report \\ Progressive Multifocal Leukoencephalopathy in a HIV Negative, Immunocompetent Patient
}

\author{
T. Nanda \\ Columbia College of Physicians and Surgeons, Columbia University Medical Center, 161 Fort Washington Avenue, \\ New York, NY 10032, USA \\ Correspondence should be addressed to T. Nanda; tn2290@columbia.edu
}

Received 23 May 2016; Revised 28 June 2016; Accepted 10 July 2016

Academic Editor: Isabella Laura Simone

Copyright (C) 2016 T. Nanda. This is an open access article distributed under the Creative Commons Attribution License, which permits unrestricted use, distribution, and reproduction in any medium, provided the original work is properly cited.

\begin{abstract}
Progressive multifocal leukoencephalopathy $(\mathrm{PML})$ is a rare demyelinating disease most common in immunodeficient patients. It occurs due to reactivation of the John Cunningham Virus (JCV) and carries a poor prognosis, with a median life expectancy of 6 months. We report a case of a 66-year-old man with a history of HCV related cirrhosis (HCV) and hepatocellular carcinoma (HCC) who was found to have PML in the setting of a negative viral load in the CSF and a CD4+>200. He initially presented with two weeks of mild confusion and word-finding difficulty concerning for hepatic encephalopathy. An MRI was notable for extensive T2/FLAIR hyperintensity signal in the left temporal lobe. Brain biopsy was positive for JCV. PML is rare in immunocompetent individuals, especially in the setting of a negative viral load. It is possible, however, that transient states of immunosuppression may have been responsible in this case. Although viral load was reported as negative, virus may still have been detected but was below the quantifiable threshold. It is important for clinicians to note that a negative result does not necessarily exclude the possibility of PML, and care should be taken to review lab values on viral load in closer detail.
\end{abstract}

\section{Introduction}

Progressive multifocal encephalopathy (PML) is a fatal demyelinating disease generally present in patients with severe immunosuppression such as HIV/AIDS, organ transplant recipients, those with hematological malignancy, and those receiving immunosuppressant therapy. PML, however, can also rarely present in immunocompetent patients, or in patients with transient or occult immunosuppression. Although limited to case reports, patients with a low to undetectable John Cunningham Virus (JCV) viral load may still present with PML. We present a patient with hepatic cirrhosis and hepatocellular carcinoma who was still found to have PML in the setting of a CD4+ count $>200$ and a negative viral load.

\section{Case Presentation}

A 66-year-old male with a past medical history of $\mathrm{HCV}$ related cirrhosis was admitted to the medicine service for altered mental status, worsening dyspnea on exertion, and paraphasic errors. His altered mental status had developed over the past two weeks and was characterized by mild confusion and word-finding difficulty. He had no other complaints and denied any recent travel or sick contacts.

On exam he was found to have some mild asterixis and word finding difficulty, often describing an object or concept rather than stating it (i.e., referring to staples as "wing things" or describing Clinton as the "future President's wife"). The rest of the exam was unremarkable with no other neurologic deficits. He was stating $97 \%$ on room air, which dropped to $92 \%$ with walking. The rest of his vitals were within normal range. Out of concern for hepatic encephalopathy he was treated with Lactulose and Rifaximin, with no improvement in mental status.

The rest of his workup was extensive. Infectious sources (blood, urine, CSF, and chest X-ray) were negative. Cardiac workup was unremarkable. An IR guided lumbar puncture showed four white blood cells with an $80 \%$ lymphocytic predominance. The rest of his CSF findings, including cytology and flow cytometry, were unremarkable. JCV viral load was reported as negative ( $<72$ copies) by polymerase chain 
reaction assay. The patient had normal complement and was HIV negative, with negative ANA, paraneoplastic panel, NMO antibodies, ACE, myeloperoxidase antibodies, and serine protease 3 . Of note, the patient's total WBC count was consistently low, ranging from 1.96 to 2.58, with an unremarkable differential.

In regard to his $\mathrm{HCV}$, he had only partial response to PEG-Interferon + Ribavirin. A recent magnetic resonance cholangiopancreatography demonstrated malignant transformation of a dysplastic nodule, now $1.8 \mathrm{~cm}$ in size.

An MRI was also obtained for possible cerebral edema related to hepatic encephalopathy. Extensive T2/FLAIR hyperintensity was found in the left temporal lobe insula and subinsula region, left frontal horn, and left lateral ventricle. There were also multiple foci of T2/FLAIR hyperintensity in the left frontal and parietal lobe. The lesions did not show diffusion restriction and there was no hemosiderin on SWI sequences. The findings were thought to be characteristic of PML.

In the setting of a negative JCV viral load in the CSF, however, other etiologies were considered, including acute disseminated encephalomyelitis, focal seizures, vasculopathy, or other inflammatory causes such as sarcoidosis, although these were considered less likely. Primary neoplasm or metastatic disease was deemed unlikely, but per neurooncologic recommendations an MRI spectroscopy was performed which did not find increased blood flow or perfusion to the regions of T2/FLAIR hyperintensity on the prior MRI. Single voxel spectroscopy of the area with T2/FLAIR hyperintensity demonstrated elevated choline and lactate consistent with increased cell membrane turnover. The differential remained broad, consistent with either an active demyelinating process or a low-grade neoplasm.

At the time, due to a lack of diagnosis, the patient underwent a left temporal brain biopsy with superior temporal artery biopsy. Biopsy cytology was unrevealing, but final pathology returned positive for JCV by a combination of immunostaining, histopathologic features, and PCR, resulting in a final diagnosis of PML. To determine immune status, a T-cell panel was ordered twice, which showed consistently reduced total $\mathrm{T}$ cells $(643,512)$ and reduced helper $\mathrm{T}$ cells $(342,268)$, with a normal CD4+/CD8+ ratio.

\section{Discussion}

JCV occurs most often in childhood as an infection without apparent illness. Antibodies are present in eighty-six percent of adults [1]. It is a latent virus, resident in the kidneys and lymphoid organs. Reactivation of latent virus in the setting of severe immunosuppression results in the development of PML. JCV reactivation is a lytic infection of oligodendrocytes, consistent with our patient's spectroscopy findings of demyelination and increased cell membrane turnover. $79 \%$ of affected individuals have AIDS [2]. The rest are divided into patients with hematological malignancies, organ transplant recipients on immunosuppressive drugs, and most recently those receiving immunomodulating drugs such as Natalizumab, Rituximab, or Efalizumab for Crohn's disease, multiple sclerosis, and other dysimmune disorders [3-5]. PML has also been found in patients receiving HAART therapy for HIV, a condition called immune reconstitution inflammatory syndrome [6]. Prognosis is very poor, with a median survival in HIV negative patients of 3 months [7].

While classic PML presents in patients with $<200$ CD4+ cells and only in 5\% of those with severe immunodeficiency [8], rare cases of PML in immunocompetent patients with and without underlying disease have been reported [9-11]. Those with underlying disease have been defined as "PML in the presence of occult or transient immunodepression," presumably caused by conditions such as hepatic cirrhosis, chronic renal failure, pregnancy, dementia, and dermatomyositis. This makes the diagnosis of PML particularly challenging, as transient states of immunodepression or immunodysfunction may not always be apparent, and the number of additional conditions ranges from idiopathic CD4+ depression to solid tumor malignancies [12].

In our patient, the possible causes of his occult immunodepression are many and likely a combination of several factors. It is known that cell mediated response, particularly CD4+ cells, is integral to the containment of JCV. Even in situations where CD4+ and CD8+ levels are normal, failures of the immune response itself may be enough to allow the development of PML. In one study of 38 reported cases of PML in patients with possible occult or transient immunosuppression by Gheuens et al. [12], cirrhosis was found in seven PML patients. Three of those had documented CD4+ lymphocytopenia or leucopenia. It is also known that patients with $\mathrm{HCV}$ or $\mathrm{HBV}$ related liver disease have a marked depression in cell mediated immune function, especially in the setting of anemia and hypoalbuminemia [13]. Our patient had consistent leucopenia, anemia, and hypoalbuminemia (range 2.6 to 2.8) in the setting of HCV liver cirrhosis. There is also some evidence of solid malignancies causing immune dysregulation via tumor-derived soluble factors such as IL10 and TGF-beta. It is possible that patient's newly malignant liver nodule played a contributing role [14].

It is also possible that patient's immune dysregulation is idiopathic in nature. Idiopathic CD4+ lymphocytopenia (ICL) is defined as a CD4+ count $<300$ or a CD $4+$ count $<20 \%$ of the total $\mathrm{T}$ cell count on two occasions, with no evidence of HIV infection, defined immunodeficiency, or immunosuppressing therapy [15]. Our patient meets all criteria, aside from a T-cell panel from two separate occasions, as he was lost to followup after discharge. ICL is a heterogeneous disease with a still unknown pathophysiology. Some studies suggest its development after an opportunistic infection, such as cryptococcosis or extrapulmonary tuberculosis [16]. Increased CD4+ cell apoptosis and a reduction in IL-7 receptors have also been implicated [17]. Bone marrow studies of patients with ICL have shown a reduction in early CD4+ stem cell precursors [18]. It is unknown if our patient ever had an opportunistic infection that may have predisposed him to developing ICL. In addition, it is difficult to determine if his immunodeficiency can instead be defined by HCC or HCV cirrhosis, as these are competing causative factors.

It is likely that an earlier and more extensive immune constitution workup would have been pursued had less 
emphasis been placed on the reportedly negative JCV viral load found in his CSF [9]. Prior cases of PML in patients with low JCV viral load have been reported, as low viral load does not correlate with the extent of disease [19]. PCR detection of JCV DNA in the CSF has been shown to have a specificity of $92 \%$ to $100 \%$ and a sensitivity of $74 \%$ to $92 \%$ when compared to brain biopsy for patients with HIV-associated PML [20]. With a $100 \%$ specificity, it is presumed that any level of virus in the CSF should be concerning for PML, with a range from undetectable to $7.71 \mathrm{log}$ copies/mL found in patients with PML in a prior study by Bossolasco et al. [21].

\section{Conclusion}

In this case, the interpretation of a negative JCV viral load in the CSF may have contributed to a delay in diagnosis. This value was reported as negative due to the inability to quantify viral load when less than 72 copies. This does not exclude the presence of virus, however. Considering the evidence of PML in patients with low to undetectable JCV titers, any level of detection should spark clinical suspicion. It is also important to consider occult or transient immunosuppressive states even with a CD $4+$ count $>200$, as this may not accurately reflect the patient's true immune status. In patients with suspected PML and no immunodeficiency, taking a detailed history on prior opportunistic infections may add to a possible diagnosis of ICL. The purpose of this discussion was to highlight the many pitfalls in evaluating a complicated neurological story for JCV reactivation, especially considering the varied and transient immunodepressive states associated with cancer or chronic disease.

\section{Competing Interests}

The author declares having no competing interests.

\section{References}

[1] T. Weber, C. Trebst, S. Frye et al., "Analysis of the systemic and intrathecal humoral immune response in progressive multifocal leukoencephalopathy," Journal of Infectious Diseases, vol. 176, no. 1, pp. 250-254, 1997.

[2] I. J. Koralnik, D. Schellingerhout, and M. P. Frosch, "Case records of the Massachusetts General Hospital. Weekly clinicopathological exercises. Case 14-2004. A 66-year-old man with progressive neurologic deficits," The New England Journal of Medicine, vol. 350, no. 18, pp. 1882-1893, 2004.

[3] B. K. Kleinschmidt-DeMasters and K. L. Tyler, "Progressive multifocal leukoencephalopathy complicating treatment with natalizumab and interferon beta-1a for multiple sclerosis," New England Journal of Medicine, vol. 353, no. 4, pp. 369-374, 2005.

[4] G. Van Assche, M. Van Ranst, R. Sciot et al., "Progressive multifocal leukoencephalopathy after natalizumab therapy for Crohn's disease," The New England Journal of Medicine, vol. 353, no. 4, pp. 362-368, 2005.

[5] E. Tavazzi, P. Ferrante, and K. Khalili, "Progressive multifocal leukoencephalopathy: an unexpected complication of modern therapeutic monoclonal antibody therapies," Clinical Microbiology and Infection, vol. 17, no. 12, pp. 1776-1780, 2011.
[6] K. Tan, R. Roda, L. Ostrow, J. McArthur, and A. Nath, "PMLIRIS in patients with HIV infection: clinical manifestations and treatment with steroids," Neurology, vol. 72, no. 17, pp. 14581464, 2009.

[7] I. J. Koralnik, "Progressive multifocal leukoencephalopathy revisited: has the disease outgrown its name?" Annals of Neurology, vol. 60, no. 2, pp. 162-173, 2006.

[8] M. W. Ferenczy, L. J. Marshall, C. D. S. Nelson et al., "Molecular biology, epidemiology, and pathogenesis of progressive multifocal leukoencephalopathy, the JC virus-induced demyelinating disease of the human brain," Clinical Microbiology Reviews, vol. 25, no. 3, pp. 471-506, 2012.

[9] P. G. Christakis, D. Okin, A. J. Huttner, and J. M. Baehring, "Progressive multifocal leukoencephalopathy in an immunocompetent patient," Journal of the Neurological Sciences, vol. 326, no. 1-2, pp. 107-110, 2013.

[10] K. K. Johansen, S. H. Torp, J. Rydland, and J. O. Aasly, "Progressive multifocal leukoencephalopathy in an immunocompetent patient?" Case Reports in Neurology, vol. 5, no. 3, pp. 149-154, 2013.

[11] V. C. Gourineni, T. Juvet, Y. Kumar, D. Bordea, and K. N. Sena, "Progressive multifocal leukoencephalopathy in a 62-yearold immunocompetent woman," Case Reports in Neurological Medicine, vol. 2014, Article ID 549271, 4 pages, 2014.

[12] S. Gheuens, G. Pierone, P. Peeters, and I. J. Koralnik, "Progressive multifocal leukoencephalopathy in individuals with minimal or occult immunosuppression," Journal of Neurology, Neurosurgery and Psychiatry, vol. 81, no. 3, pp. 247-254, 2010.

[13] A. A. Wahib, A. A. Masoud, A. A. Halem et al., "Cell mediated immune response in chronic liver diseases: schistosomal, viral, and neoplastic," Journal of the Egyptian Society of Parasitology, vol. 28, pp. 929-939, 1998.

[14] R. Kim, M. Emi, and K. Tanabe, "Cancer immunosuppression and autoimmune disease: beyond immunosuppressive networks for tumour immunity," Immunology, vol. 119, no. 2, pp. 254-264, 2006.

[15] S. Haider, D. Nafziger, J. A. Gutierrez, I. Brar, N. Mateo, and J. Fogle, "Progressive multifocal leukoencephalopathy and idiopathic CD4+ lymphocytopenia: a case report and review of reported cases," Clinical Infectious Diseases, vol. 31, no. 4, pp. E20-E22, 2000.

[16] D. Zonios, J. Falloon, J. Bennett et al., "Idiopathic CD4 ${ }^{+}$lymphocytopenia: natural history and prognostic factors," Blood, vol. 112, no. 2, pp. 287-294, 2008.

[17] P. Hubert, F. Bergeron, V. Ferreira et al., "Defective p56 $6^{\text {Lck }}$ activity in T cells from an adult patient with idiopathic $\mathrm{CD} 4^{+}$ lymphocytopenia," International Immunology, vol. 12, no. 4, pp. 449-457, 2000.

[18] A. Isgrò, M. C. Sirianni, C. Gramiccioni, I. Mezzaroma, A. Fantauzzi, and F. Aiuti, "Idiopathic CD4+ lymphocytopenia may be due to decreased bone marrow clonogenic capability," International Archives of Allergy and Immunology, vol. 136, no. 4, pp. 379-384, 2005.

[19] D. García de Viedma, M. Díaz Infantes, P. Miralles et al., "JC virus load in progressive multifocal leukoencephalopathy: analysis of the correlation between the viral burden in cerebrospinal fluid, patient survival, and the volume of neurological lesions," Clinical Infectious Diseases, vol. 34, no. 12, pp. 1568-1575, 2002.

[20] I. J. Koralnik, D. Boden, V. X. Mai, C. I. Lord, and N. L. Letvin, "JC virus DNA load in patients with and without progressive 
multifocal leukoencephalopathy," Neurology, vol. 52, no. 2, pp. 253-260, 1999.

[21] S. Bossolasco, G. Calori, F. Moretti et al., "Prognostic significance of JC virus DNA levels in cerebrospinal fluid of patients with HIV-associated progressive multifocal leukoencephalopathy," Clinical Infectious Diseases, vol. 40, no. 5, pp. 738-744, 2005. 


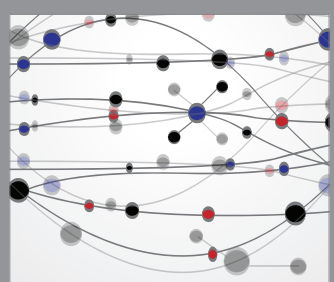

The Scientific World Journal
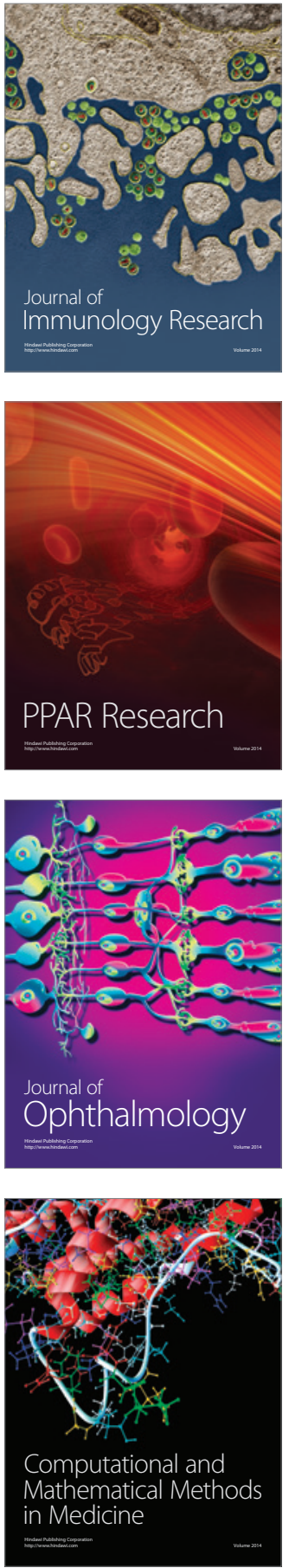

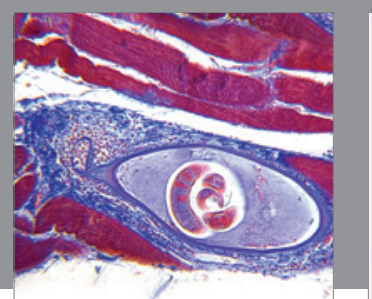

Gastroenterology Research and Practice

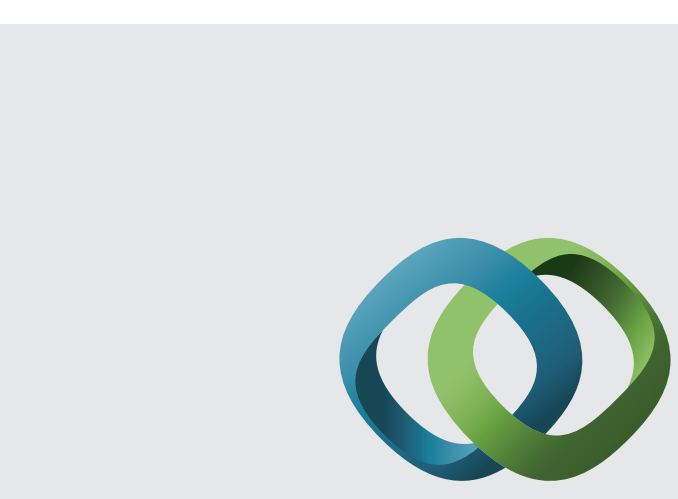

\section{Hindawi}

Submit your manuscripts at

http://www.hindawi.com
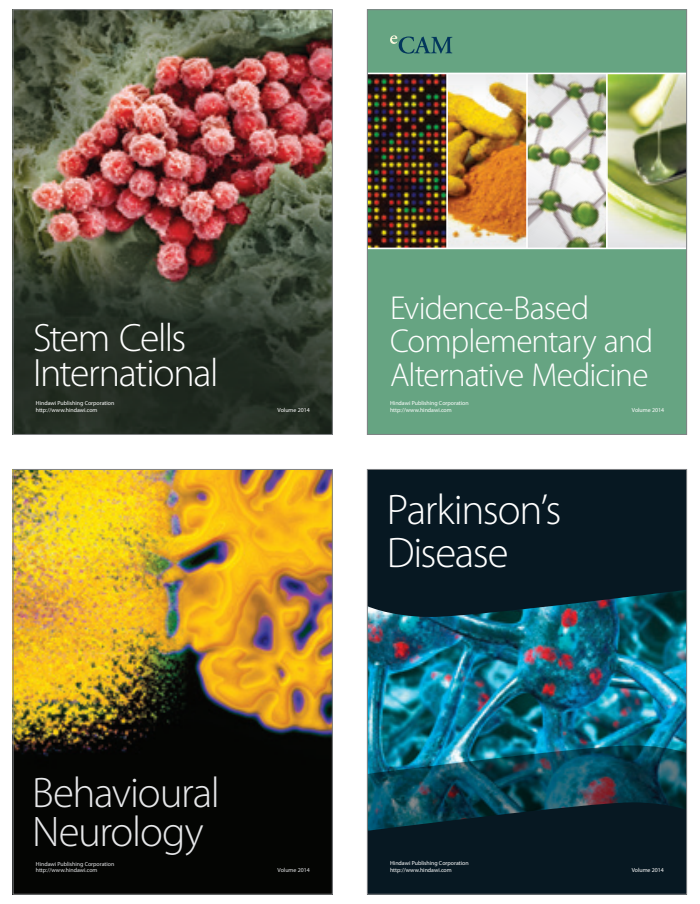
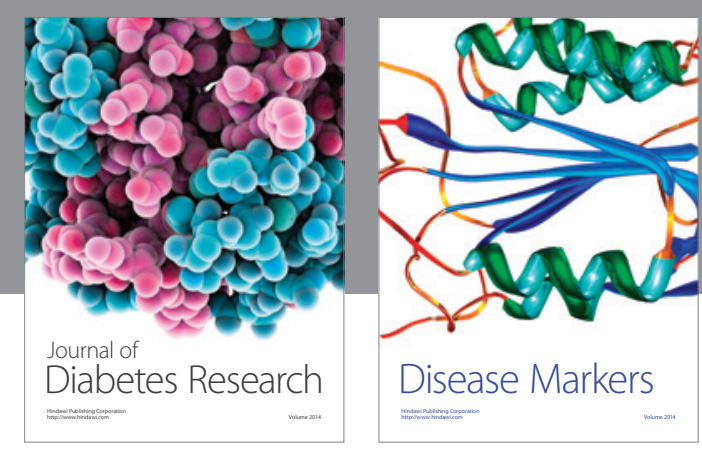

Disease Markers
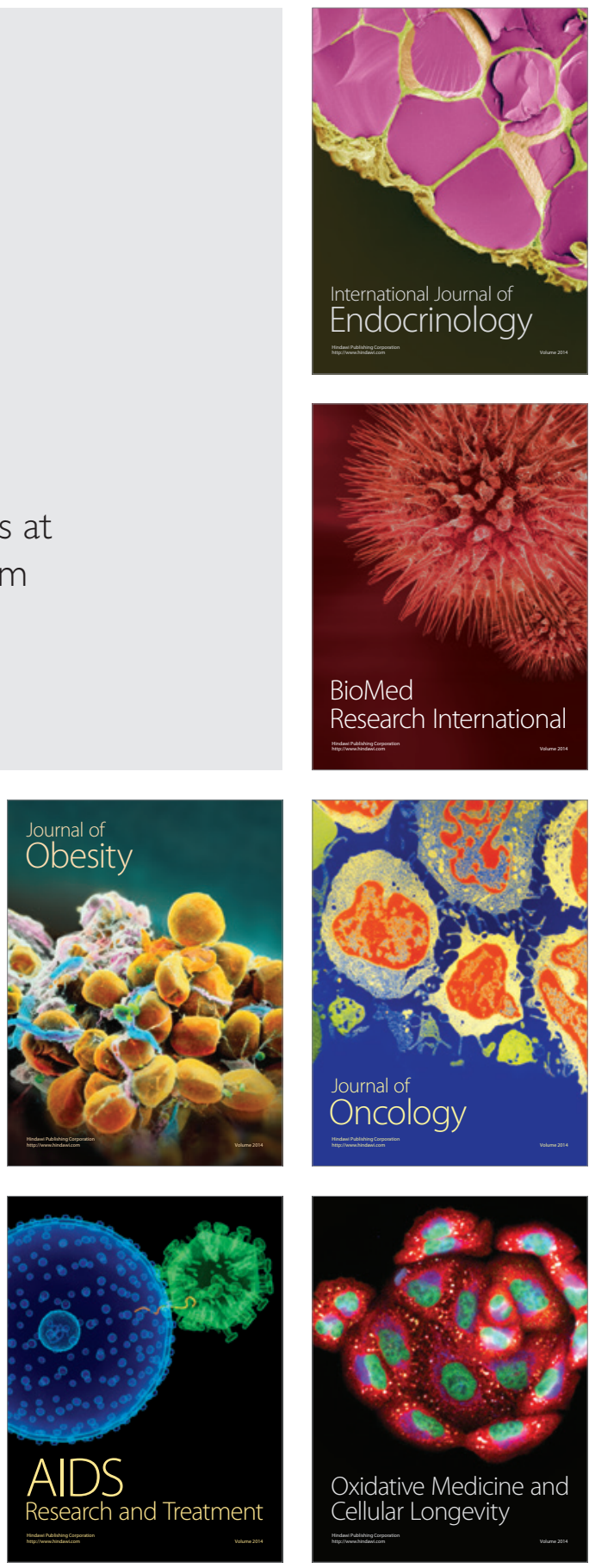